\title{
Cold Stress Evaluation among Maize (Zea mays L.) Inbred Lines in Different Temperature Conditions
}

\author{
Muhammad Qudrat Ullah Farooqi, Ju Kyong Lee* \\ Department of Applied Plant Sciences, College of Agriculture and Life Sciences, Kangwon National University, Chuncheon 24341, \\ Korea
}

\begin{abstract}
Maize (Zea mays L.) is a crop in a tropical region which resists growing under sensitive temperature. This study was conducted to evaluate the performance of Canadian maize inbred lines under controlled cold stress conditions $\left(5^{\circ} \mathrm{C}, 10^{\circ} \mathrm{C}\right.$, and $\left.23^{\circ} \mathrm{C}\right)$. Data were recorded by measuring germination rate, index, root length, and seed vigour index values. Five higher and three lower tolerant inbred lines were shortlisted. The data were analyzed using analysis of variance, while mean values were compared using Tukey's Honest Significant Difference Test at $\alpha=0.05$ and at $\alpha=0.01$. Using Genstat software, correlation was done. A strong correlation $(P<0.05)$ was found between germination rate and germination index under all stress conditions. Root length and vigour index were also strongly correlated with germination rate under $5^{\circ} \mathrm{C}$ stress condition and compared to $10^{\circ} \mathrm{C}$ and $23^{\circ} \mathrm{C}$ stress conditions. Our results suggested that five (CO439, CO438, CO450, CO435, and CO445) among 22 maize inbred lines performed better under $5^{\circ} \mathrm{C}$ cold stress condition and thus had the potential to develop maize hybrids to increase grain yield under environmentally stressful conditions in South Korea.
\end{abstract}

Keywords Cold tolerance, Germination, Vigour, Maize, Inbred line

\section{INTRODUCTION}

Crop plants respond to environmental changes by modifying their pattern of gene expression and products under stressful conditions for survival and yield (Sanghera et al. 2011). Mirosavljević et al. (2013) found that root weight, shoot weight, root length (RL), shoot length, germination energy, and rate were affected due to the stress in plants. Hence, tolerance of crop plants is essential for vigourous growth and for resisting insects and pests. Thus, for good yield and to withstand insects and pests, crops should be resistant against various abiotic and biotic factors. Chilling temperature is one of the important constraints during early seedling growth for global crop (maize) production (Guan et al. 2009; Ali et al. 2015a). Cold stress can influence periodic life cycle and productivity of crop plant in the early developmental stages. Chilling stress basically disturbs mean germination time
(MGT) among cultivars; this is significantly correlated with the shoot length and dry weight of plant. Furthermore, low temperature affects photosynthetic rate, secondary osmotic, and oxidative stress which lead to cell damage (Matthews et al. 2011; Riva-Roveda and Périlleux 2015).

Maize (Zea mays L.) is highly sensitive to low temperature due to their tropical origin. Abiotic stresses cause adverse effect in growth, physiology, and biochemical processes (Ali et al. 2015b). Nowadays, maize crop is cultivated in a wide range of altitudes. Many adaptations have been implemented to keep its high production yield (Ali et al. 2014). In maize plants, cold stress germination traits are important to check for earlier seasonal adaptability (Hoffman et al. 2015). Revilla et al. (2014) revealed that the cold tolerance of maize is an important investigation factor for the vegetative period extension. Matthews et al. (2011) suggested that, compared with other germination tests, cold test is better in assessing early

Received August 12, 2016; Revised August 16, 2016; Accepted August 17, 2016; Published August 31, 2016

*Comesponding author Ju Kyong Lee, jukyonglee@kangwon.ac.kr, Tel: +82-33-250-6415, Fax: +82-33-255-5558 
growth vigour. Among the maize inbred lines, there is great variability for the cold tolerance evaluation under certain environmental conditions. Germination under any kind of stress is important to assume the vigour of that plant (Alvarez et al. 2014). Cold tolerance is a complex study to investigate; however, molecular biology and physiological approaches are the best ways to evaluate the low temperature response of maize at sprouting stage (Bano et al. 2015). Seedling vigour and RL are directly influenced with environmental stress.

Furthermore, seed storage is a big issue under various climatic conditions. For seed storage to ensure quality, lower temperature is better than high temperature. Thus, evaluation under cold stress is essential. Chilling temperature halts membrane permeability, photosynthetic rate, and osmotic potential in plant; at high temperature conditions, seed deterioration increases due to high moisture acceleration (Abba and Lovato 1999). In cold tolerance, multiple genes are involved to control chilling stress. The quality of maize seed in chilling temperature can be negatively affected. However, the genotype with compact root system has good metabolic activities and ability to resist against harsh conditions. It can sprout better seedling and more vigour (DeVries et al. 2007). The cold storage of seed prevents pest/insect attack and helps seeds resist microorganisms. Seed quality becomes affected when moisture content is higher than 14\% (Govender et al. 2008). At low temperature, plants respond to alterations in the expression of gene and protein product pattern. Genes can figure out, through germination testing, which are responsible for stress response during the germination of crop plant.

At cold temperature, physiological damage can reduce seed vigour and viability (DeVries et al. 2007). However, different genotypes have different levels of survival under cold environment as their genetic makeup can either endure or is not tolerant against freezing temperature. The best evaluation of maize germination can be done when a plant shows rapid germination and vigour, and resists insect pests under low temperature stress. The seed vigour and viability loss are greater in decreasing temperature or abrupt changes in environmental conditions (TeKrony and Hunter 1994; Woltz et al. 2006). Tekrony (2003) revealed from his experimentation that seed vigour test is better for the evaluation of physiological seed quality. Crop yield reduction is indirectly related with low seed vigour. During cold germination, the significant difference values of root and shoot among different genotypes were exhibited.

Hence, seed vigour and viability directly impact the crop yield and performance of seed quality under certain conditions. The increase or decrease of yield in crop plants is directly associated with seed vigour. Thus, germination test is necessary to check the performance of different cultivars before cultivation. This study's results demonstrate that study maize inbred lines have the potential to develop maize hybrids in cold climatic condition such as in South Korea. In detail, our aims to conduct this experiment were:

i) To identify the seed vigour, germination, and seedling growth of maize inbred lines in different temperature conditions.

ii) To check early germination and establishment associated with important physiological and phonological characters.

iii) To screen out the diversified pool of maize germplasm that can tolerate chilling temperature and differentiate high and low tolerant inbred lines, which will be further experimented in the molecular breeding program.

\section{MATERIALS AND METHODS}

\section{Seed material}

A total of 22 maize inbred lines, developed at the Institute of Eastern Cereal and Oilseed Research Centre in Canada, were used in this study (Table 1). Seeds for each inbred line were soaked in water before going to a germination test. All seeds were treated with Captan (cis-N trichloromethylthio-4-cyclohexene-1, 2-dicarboximide).

\section{Cold germination test}

Testing was carried out in a growth chamber in three replicates with 20 seeds of each inbred line per replicate. Seeds were planted on petri dishes with moist filter paper and placed in growth chambers at constants of $5^{\circ} \mathrm{C}, 10^{\circ} \mathrm{C}$, 
Table 1. Derivations of Canadian maize inbred lines used in this study.

\begin{tabular}{|c|c|c|}
\hline Inbred/Autogame & Derivation/Source & Heterotic group \\
\hline $\mathrm{CO} 430$ & Fusarium Resistant Synthetic & P3990 \\
\hline $\mathrm{CO} 431$ & Fusarium Resistant Synthetic & Iodent \\
\hline $\mathrm{CO} 432$ & Fusarium Resistant Synthetic C1 & Minn13 \\
\hline $\mathrm{CO} 433$ & Pride K127 & Minn 13 \\
\hline $\mathrm{CO} 434$ & $\mathrm{CM} 105 \times \mathrm{A} 632$ & BSSS \\
\hline $\mathrm{CO} 435$ & $\mathrm{~A} 632 \times \mathrm{A} 634$ & BSSS \\
\hline $\mathrm{CO} 436$ & $\mathrm{CO} 275 \times \mathrm{CO} 300$ & P3994 \\
\hline $\mathrm{CO} 437$ & European Synthetic & E.Flint \\
\hline $\mathrm{CO} 438$ & $\mathrm{CB} 3 \times \mathrm{CL} 29$ & P3994 \\
\hline $\mathrm{CO} 439$ & Nebraska BSSS & BSSS \\
\hline $\mathrm{CO} 440$ & Pride $5 \times \mathrm{CO} 258 \mathrm{~V}$ & Minn 13 \\
\hline $\mathrm{CO} 441$ & Jacques $7700 \times \mathrm{CO} 298$ & Lanc \\
\hline $\mathrm{CO} 442$ & Iodent/NSS & Iodent \\
\hline $\mathrm{CO} 443$ & $\mathrm{~B} 104 \times \mathrm{CO} 272$ & BSSS/E.Butler \\
\hline $\mathrm{CO} 444$ & $\mathrm{~S} 1381 \times \mathrm{CO} 382$ & E.Flint \\
\hline $\mathrm{CO} 445$ & $\mathrm{CO} 386 \times \mathrm{W} 64 \mathrm{AHt}$ & Lanc \\
\hline $\mathrm{CO} 446$ & $\mathrm{CO} 341 \times \mathrm{CO} 328$ & BSSS \\
\hline $\mathrm{CO} 447$ & $\mathrm{CO} 352 \times \mathrm{CO} 328$ & BSSS/Minn \\
\hline $\mathrm{CO} 448$ & $\mathrm{CO} 273 \times \mathrm{CO} 431$ & P3990/Iodent \\
\hline $\mathrm{CO} 449$ & $\mathrm{CO} 432 \times \mathrm{CO} 433$ & Minn13 \\
\hline $\mathrm{CO} 450$ & Eyespot Resistant Synthetic (99ESR) & BSSS/Mix \\
\hline $\mathrm{CO} 451$ & $\mathrm{CO} 309 \times \mathrm{CO} 328$ & BSSS/Minn \\
\hline
\end{tabular}

and $23^{\circ} \mathrm{C}$ under completely randomized design. Five $\mathrm{ml}$ of distilled water was added at the beginning of the experiment and every two days during the duration of the study. The number of germinated seeds was recorded daily for 7 days. Seeds were considered germinated when the radicle was $2 \mathrm{~mm}$ long. Evaluations of germination were made every day. Seeds were tested in chilling temperature under International Seed Testing Association (ISTA) (2002) seed testing rules.

\section{Germination analysis}

Germination rate (GR): The GR (\%) of seeds of each inbred line was calculated with the appropriate formula:

GR $(\%)=($ number of seeds sprouted/total number of seeds sprouted) $\times 100$

Germination index (GI): GI and MGT were calculated using the following formula:

$\mathrm{GI}=\Sigma(\mathrm{Gt} / \mathrm{Tt})$, where $\mathrm{Gt}$ is the number of seeds germinated on each day $(\mathrm{t})$, and $\mathrm{Tt}$ is the total number of days for germination.
$\mathrm{MGT}=\sum \mathrm{Ti} \mathrm{Ni} / \sum \mathrm{Ni}$, where $\mathrm{Ni}$ is the number of newly germinated seeds at time Ti (Ruan et al. 2002).

RL: Root growth was evaluated by hand using a ruler in $\mathrm{mm}$. All three temperature treatments were separately evaluated in the same set of seeds.

Seed vigour index (SVI): Using the formula of Abdul-Baki and Anderson (1973), SVI was calculated.

$\mathrm{SVI}=($ Seedling emergence rate $\times \mathrm{RL}[\mathrm{mm}]) / 100$

\section{Statistical analysis}

The statistical analysis was performed using Microsoft Excel (2007; Microsoft, Redmond, WA, USA) and Statistical Tools for Agricultural Research (STAR), while analysis of variance (ANOVA) was conducted using SAS 9.3 software (SAS Institute, Cary, NC, USA). Means were compared using Tukey's Honest Significant Difference (HSD) Test at $\alpha=0.05$ and at $\alpha=0.01$. The correlation analysis was done using statistical software (Genstat; VSN International, Hemel Hempstead, UK). 


\section{RESULTS}

\section{Effect of germination status in different temperature conditions}

A total of 22 maize inbred lines were investigated at three different temperatures $\left(5^{\circ} \mathrm{C}, 10^{\circ} \mathrm{C}\right.$, and $23^{\circ} \mathrm{C}$, respectively). The traits were compared with least significant difference values. At lowest temperature $\left(5^{\circ} \mathrm{C}\right)$ treatment, the highest rate of germination was shown in $\mathrm{CO} 439$ inbred line. The $\mathrm{CO} 451$ and $\mathrm{CO} 450$ inbred lines showed the highest GR at $10^{\circ} \mathrm{C}$ treatment. Almost, all inbred lines showed higher GR at $23^{\circ} \mathrm{C}$ condition, which was almost near room temperature. In variation, the GR of $\mathrm{CO} 450$, $\mathrm{CO} 435, \mathrm{CO} 444$, and $\mathrm{CO} 451$ were relatively higher at $10^{\circ} \mathrm{C}$ as compared to $23^{\circ} \mathrm{C}$ (Table 2). As a result, four inbred lines, namely $\mathrm{CO} 439, \mathrm{CO} 438, \mathrm{CO} 450, \mathrm{CO} 435$, and $\mathrm{CO} 445$ (weaker only at $10^{\circ} \mathrm{C}$ ), exhibited a high GR of $70 \%$ at different temperatures $\left(5^{\circ} \mathrm{C}, 10^{\circ} \mathrm{C}\right.$, and $\left.23^{\circ} \mathrm{C}\right)$. Whereas, three inbred lines, namely CO437, CO436, and CO440, showed the lowest GR under the above given germination parameters in the control condition. The rest of the maize inbred lines showed medium GR, GI, RL, and SVI.

At lowest temperature $\left(5^{\circ} \mathrm{C}\right)$ treatment, the highest and lowest values of GI were observed in $\mathrm{CO} 438$ and $\mathrm{CO} 440$, respectively (Table 3). At $10^{\circ} \mathrm{C}, \mathrm{CO} 450$ showed the highest value of GI, and CO436 showed the lowest value of GI. At $23^{\circ} \mathrm{C}$, most inbred lines showed comparatively high GI, except for two inbred lines (CO443 and CO437). The longest RL was observed in $\mathrm{CO} 433$, and the shortest RL was observed in $\mathrm{CO} 440$ at $5^{\circ} \mathrm{C}$ treatment (Table 4). At $10^{\circ} \mathrm{C}, \mathrm{CO} 444$ showed the longest $\mathrm{RL}$, and $\mathrm{CO} 436$ showed the shortest RL. At $23^{\circ} \mathrm{C}, \mathrm{CO} 441$ showed the longest RL, and $\mathrm{CO} 436$ showed the shortest RL.

In addition, Table 5 demonstrates the SVI data at different temperatures of different inbred lines. The strongest SVI was observed in $\mathrm{CO} 439$, and the weakest SVI was

Table 2. Germination rate of 22 maize inbred lines in different temperature conditions.

\begin{tabular}{|c|c|c|c|c|}
\hline \multirow{2}{*}{ Inbred lines } & \multicolumn{3}{|c|}{ Germination rate $(\%)$ at different temperature conditions } & \multirow{2}{*}{ Characters } \\
\hline & $5^{\circ} \mathrm{C}$ & $10^{\circ} \mathrm{C}$ & $23^{\circ} \mathrm{C}$ & \\
\hline $\mathrm{CO} 439$ & $90.0 \mathrm{a}^{\mathrm{z})}$ & $98.3 \mathrm{a}$ & $98.3 \mathrm{a}$ & High tolerant \\
\hline CO438 & $88.3 \mathrm{a}$ & $81.6 \mathrm{abc}$ & $90.0 \mathrm{abc}$ & High tolerant \\
\hline $\mathrm{CO} 450$ & $85.0 \mathrm{ab}$ & $100.0 \mathrm{a}$ & $98.3 \mathrm{a}$ & High tolerant \\
\hline $\mathrm{CO} 435$ & $80.0 \mathrm{abc}$ & $98.3 \mathrm{a}$ & $91.6 \mathrm{abc}$ & High tolerant \\
\hline $\mathrm{CO} 445$ & 75.0abcd & $45.0 \mathrm{ef}$ & $91.6 \mathrm{abc}$ & High tolerant \\
\hline $\mathrm{CO} 444$ & $65.0 \mathrm{bcde}$ & $95.0 \mathrm{a}$ & $98.3 \mathrm{a}$ & Medium tolerant \\
\hline $\mathrm{CO} 433$ & $65.0 \mathrm{bcde}$ & $90.0 \mathrm{ab}$ & $93.3 \mathrm{ab}$ & Medium tolerant \\
\hline $\mathrm{CO} 447$ & $61.6 \mathrm{cde}$ & $76.6 \mathrm{abcd}$ & $96.6 \mathrm{ab}$ & Medium tolerant \\
\hline $\mathrm{CO} 431$ & $60.0 \mathrm{cdef}$ & $86.6 \mathrm{ab}$ & $90.0 \mathrm{abc}$ & Medium tolerant \\
\hline $\mathrm{CO} 451$ & 55.0defg & $100.0 \mathrm{a}$ & $90.0 \mathrm{abc}$ & Medium tolerant \\
\hline $\mathrm{CO} 442$ & $53.3 \mathrm{efgh}$ & $65.0 \mathrm{bcdef}$ & $96.6 \mathrm{ab}$ & Medium tolerant \\
\hline $\mathrm{CO} 443$ & 40.0fghi & $50.0 \mathrm{def}$ & $66.6 \mathrm{e}$ & Medium tolerant \\
\hline CO449 & $36.6 \mathrm{ghi}$ & $88.3 \mathrm{ab}$ & $93.3 \mathrm{ab}$ & Medium tolerant \\
\hline $\mathrm{CO} 441$ & $33.3 \mathrm{hi}$ & $40.0 \mathrm{fg}$ & $91.6 \mathrm{abc}$ & Medium tolerant \\
\hline CO446 & $33.3 \mathrm{hi}$ & $43.3 \mathrm{~g}$ & $100.0 \mathrm{a}$ & Medium tolerant \\
\hline $\mathrm{CO} 432$ & $31.6 \mathrm{i}$ & $90.0 \mathrm{ab}$ & $91.6 \mathrm{abc}$ & Medium tolerant \\
\hline $\mathrm{CO} 434$ & $30.0 \mathrm{i}$ & $88.3 \mathrm{ab}$ & $100.0 \mathrm{a}$ & Medium tolerant \\
\hline CO430 & $23.3 \mathrm{ij}$ & 73.3abcde & $73.3 \mathrm{de}$ & Medium tolerant \\
\hline CO448 & $23.3 \mathrm{ij}$ & $93.3 \mathrm{ab}$ & $88.3 \mathrm{abc}$ & Medium tolerant \\
\hline $\mathrm{CO} 437$ & $8.3 \mathrm{jk}$ & $65.0 \mathrm{bcdef}$ & $80.0 \mathrm{~cd}$ & Low tolerant \\
\hline CO436 & $3.3 \mathrm{jk}$ & $11.6 \mathrm{~g}$ & $85.0 \mathrm{bcd}$ & Low tolerant \\
\hline $\mathrm{CO} 440$ & $1.6 \mathrm{k}$ & $55.0 \mathrm{cdef}$ & $93.3 \mathrm{ab}$ & Low tolerant \\
\hline
\end{tabular}

\footnotetext{
${ }^{\mathrm{z})}$ Means in the same column followed by different letters are significantly different according to Tukey's New Multiple
} Range test $(P<0.05)$. 
Table 3. Germination index of 22 maize inbred lines in different temperature conditions.

\begin{tabular}{|c|c|c|c|c|}
\hline \multirow{2}{*}{ Inbred lines } & \multicolumn{3}{|c|}{ Germination index at different temperature conditions } & \multirow{2}{*}{ Characters } \\
\hline & $5^{\circ} \mathrm{C}$ & $10^{\circ} \mathrm{C}$ & $23^{\circ} \mathrm{C}$ & \\
\hline CO439 & $1.7 \mathrm{a}^{\mathrm{z})}$ & $2.2 \mathrm{ab}$ & 7.4abcd & High tolerant \\
\hline $\mathrm{CO} 438$ & $1.8 \mathrm{a}$ & $1.6 \mathrm{bcde}$ & $6.2 \mathrm{cde}$ & High tolerant \\
\hline $\mathrm{CO} 450$ & $1.6 \mathrm{a}$ & $2.3 \mathrm{a}$ & $7.8 \mathrm{abc}$ & High tolerant \\
\hline $\mathrm{CO} 435$ & $1.5 \mathrm{ab}$ & $2.0 \mathrm{abc}$ & 7.3abcd & High tolerant \\
\hline $\mathrm{CO} 445$ & $1.5 \mathrm{abc}$ & $1.0 \mathrm{fg}$ & 7.3abcd & High tolerant \\
\hline $\mathrm{CO} 444$ & $1.2 \mathrm{bcd}$ & $2.0 \mathrm{abc}$ & $6.6 \mathrm{bcd}$ & Medium tolerant \\
\hline $\mathrm{CO} 433$ & $1.2 \mathrm{bcd}$ & $2.0 \mathrm{abc}$ & 7.4abcd & Medium tolerant \\
\hline $\mathrm{CO} 447$ & $1.2 \mathrm{bcd}$ & $1.6 \mathrm{bcde}$ & $7.5 \mathrm{abc}$ & Medium tolerant \\
\hline $\mathrm{CO} 431$ & $1.1 \mathrm{~cd}$ & 1.7abce & $6.4 \mathrm{~cd}$ & Medium tolerant \\
\hline $\mathrm{CO} 451$ & $1.0 \mathrm{def}$ & $2.0 \mathrm{abc}$ & $7.0 \mathrm{bcd}$ & Medium tolerant \\
\hline $\mathrm{CO} 442$ & $1.1 \mathrm{de}$ & $1.5 \mathrm{cdef}$ & 7.4abcd & Medium tolerant \\
\hline CO443 & 0.7 efg & $1.0 \mathrm{fg}$ & $4.7 \mathrm{e}$ & Medium tolerant \\
\hline $\mathrm{CO} 449$ & 0.7 efg & $1.8 \mathrm{abcd}$ & 7.3abcd & Medium tolerant \\
\hline $\mathrm{CO} 441$ & $0.6 \mathrm{fg}$ & $0.8 \mathrm{gh}$ & 7.1abcd & Medium tolerant \\
\hline $\mathrm{CO} 446$ & $0.6 \mathrm{fg}$ & $1.0 \mathrm{fg}$ & $8.0 \mathrm{ab}$ & Medium tolerant \\
\hline $\mathrm{CO} 432$ & $0.6 \mathrm{fg}$ & $2.0 \mathrm{abc}$ & $7.7 \mathrm{abc}$ & Medium tolerant \\
\hline $\mathrm{CO} 434$ & $0.6 \mathrm{~g}$ & $1.8 \mathrm{abcde}$ & $8.7 \mathrm{a}$ & Medium tolerant \\
\hline $\mathrm{CO} 430$ & $0.4 \mathrm{gh}$ & $1.5 \mathrm{cdef}$ & $7.0 \mathrm{bcd}$ & Medium tolerant \\
\hline CO448 & $0.4 \mathrm{gh}$ & $2.0 \mathrm{abc}$ & $6.2 \mathrm{cde}$ & Medium tolerant \\
\hline $\mathrm{CO} 437$ & $0.1 \mathrm{hi}$ & $1.3 \mathrm{defg}$ & $5.8 \mathrm{de}$ & Low tolerant \\
\hline $\mathrm{CO} 436$ & $0.1 \mathrm{hi}$ & $0.3 \mathrm{~h}$ & $6.5 \mathrm{bcd}$ & Low tolerant \\
\hline $\mathrm{CO} 440$ & $0.03 \mathrm{i}$ & $1.2 \mathrm{efg}$ & $7.8 \mathrm{abc}$ & Low tolerant \\
\hline
\end{tabular}

${ }^{z)}$ Means in the same column followed by different letters are significantly different according to Tukey's new multiple range test $(P<0.05)$.

Table 4. Root length of 22 maize inbred lines in different temperature conditions.

\begin{tabular}{|c|c|c|c|c|}
\hline \multirow{2}{*}{ Inbred lines } & \multicolumn{3}{|c|}{ Root length $(\mathrm{mm})$ at different temperature conditions } & \multirow{2}{*}{ Characters } \\
\hline & $5^{\circ} \mathrm{C}$ & $10^{\circ} \mathrm{C}$ & $23^{\circ} \mathrm{C}$ & \\
\hline $\mathrm{CO} 439$ & $83.6 a b^{z)}$ & 55.0def & $54.3 \mathrm{bcdefg}$ & High tolerant \\
\hline $\mathrm{CO} 438$ & 50.6def & 47.6defgh & 57.6abcdeg & High tolerant \\
\hline $\mathrm{CO} 450$ & $56.0 \mathrm{cdef}$ & $75.3 \mathrm{abc}$ & 60.3abcdef & High tolerant \\
\hline $\mathrm{CO} 435$ & $71.6 \mathrm{abcd}$ & 46.0defgh & $67.0 \mathrm{abcd}$ & High tolerant \\
\hline $\mathrm{CO} 445$ & 41.3efghi & 51.0defg & 67.0abcd & High tolerant \\
\hline $\mathrm{CO} 444$ & $86.0 \mathrm{ab}$ & $81.0 \mathrm{a}$ & 63.3abcde & Medium tolerant \\
\hline $\mathrm{CO} 433$ & $95.0 \mathrm{a}$ & 37.6fghi & 57.0abcdefg & Medium tolerant \\
\hline $\mathrm{CO} 447$ & $58.0 \mathrm{cdef}$ & $57.6 \mathrm{~cd}$ & $72.0 \mathrm{ab}$ & Medium tolerant \\
\hline $\mathrm{CO} 431$ & $76.3 \mathrm{abc}$ & 51.3defg & $52.0 \mathrm{cdefg}$ & Medium tolerant \\
\hline $\mathrm{CO} 451$ & $51.3 \mathrm{def}$ & $57.3 \mathrm{cde}$ & $50.0 \mathrm{cdefg}$ & Medium tolerant \\
\hline $\mathrm{CO} 442$ & $65.0 \mathrm{bcde}$ & 63.6abcd & 49.6cdefgh & Medium tolerant \\
\hline $\mathrm{CO} 443$ & 36.6fghi & $57.0 \mathrm{cde}$ & $45.6 \mathrm{efg}$ & Medium tolerant \\
\hline $\mathrm{CO} 449$ & 48.6defg & $61.0 \mathrm{bcd}$ & $68.0 \mathrm{abc}$ & Medium tolerant \\
\hline $\mathrm{CO} 441$ & 49.6def & 50.6defg & $73.6 \mathrm{a}$ & Medium tolerant \\
\hline $\mathrm{CO} 446$ & 71.3abcd & 36.6fghi & $47.3 \mathrm{efg}$ & Medium tolerant \\
\hline $\mathrm{CO} 432$ & 61.6bcde & $34.3 \mathrm{ghi}$ & $47.3 \mathrm{efg}$ & Medium tolerant \\
\hline $\mathrm{CO} 434$ & $45.3 \mathrm{efg}$ & 29.0hi & $42.0 \mathrm{fg}$ & Medium tolerant \\
\hline $\mathrm{CO} 430$ & 49.6def & $79.0 \mathrm{ab}$ & $60.0 \mathrm{abcdef}$ & Medium tolerant \\
\hline $\mathrm{CO} 448$ & $43.3 \mathrm{efgh}$ & $77.6 \mathrm{ab}$ & 49.6cdefgh & Medium tolerant \\
\hline $\mathrm{CO} 437$ & 18.6hi & $56.6 \mathrm{cde}$ & 63.0abcde & Low tolerant \\
\hline $\mathrm{CO} 436$ & 24.0ghi & $25.3 \mathrm{i}$ & $40.0 \mathrm{~g}$ & Low tolerant \\
\hline $\mathrm{CO} 440$ & $17.6 \mathrm{i}$ & 38.6efghi & 48.6defg & Low tolerant \\
\hline
\end{tabular}

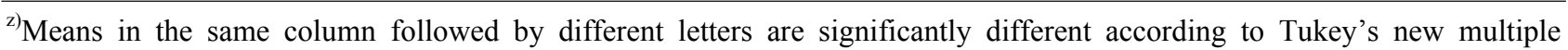
range test $(P<0.05)$. 
Table 5. Seed vigour index of 22 maize inbred lines in different temperature conditions.

\begin{tabular}{|c|c|c|c|c|}
\hline \multirow{2}{*}{ Inbred lines } & \multicolumn{3}{|c|}{ Seed vigour index at different temperature conditions } & \multirow{2}{*}{ Characters } \\
\hline & $5^{\circ} \mathrm{C}$ & $10^{\circ} \mathrm{C}$ & $23^{\circ} \mathrm{C}$ & \\
\hline CO439 & $74.5 \mathrm{a}^{\mathrm{z})}$ & $54.1 \mathrm{abcd}$ & 53.5abcdefg & High tolerant \\
\hline $\mathrm{CO} 438$ & $44.8 \mathrm{bcde}$ & 38.0bcefg & $37.0 \mathrm{gh}$ & High tolerant \\
\hline $\mathrm{CO} 450$ & $47.3 \mathrm{bcde}$ & $75.3 \mathrm{a}$ & 59.4abcde & High tolerant \\
\hline $\mathrm{CO} 435$ & $57.8 \mathrm{abc}$ & 45.0bcefgh & $36.9 \mathrm{gh}$ & High tolerant \\
\hline $\mathrm{CO} 445$ & 31.4efghi & $23.2 \mathrm{fgh}$ & $62.2 \mathrm{abcde}$ & High tolerant \\
\hline $\mathrm{CO} 444$ & $55.7 \mathrm{abcd}$ & $77.1 \mathrm{a}$ & 62.1abcde & Medium tolerant \\
\hline $\mathrm{CO} 433$ & $61.9 \mathrm{ab}$ & $34.1 \mathrm{cdefg}$ & 52.2abcdefg & Medium tolerant \\
\hline $\mathrm{CO} 447$ & $36.5 \mathrm{defgh}$ & 44.9bcdefg & $69.6 \mathrm{a}$ & Medium tolerant \\
\hline $\mathrm{CO} 431$ & 45.6bcdef & 44.5 bcdefg & 47.1defgh & Medium tolerant \\
\hline $\mathrm{CO} 451$ & 27.6fghij & $57.3 \mathrm{abc}$ & 44.8efgh & Medium tolerant \\
\hline $\mathrm{CO} 442$ & $39.5 \mathrm{cdefg}$ & 47.4bcdef & $48.0 \mathrm{cdefgh}$ & Medium tolerant \\
\hline $\mathrm{CO} 443$ & $15.7 \mathrm{ijk}$ & $27.4 \mathrm{efgh}$ & 49.9bcdefgh & Medium tolerant \\
\hline CO449 & 19.1hijk & $53.8 \mathrm{abcd}$ & 65.7abcd & Medium tolerant \\
\hline $\mathrm{CO} 441$ & $16.3 \mathrm{ijk}$ & $19.7 \mathrm{gh}$ & $67.8 \mathrm{ab}$ & Medium tolerant \\
\hline $\mathrm{CO} 446$ & 23.3ghij & $19.9 \mathrm{gh}$ & 47.3defgh & Medium tolerant \\
\hline $\mathrm{CO} 432$ & 19.5hijk & $30.5 \mathrm{defg}$ & $44.2 \mathrm{efgh}$ & Medium tolerant \\
\hline $\mathrm{CO} 434$ & $13.9 \mathrm{ijk}$ & $25.5 \mathrm{fgh}$ & $67.0 \mathrm{abc}$ & Medium tolerant \\
\hline $\mathrm{CO} 430$ & $12.4 \mathrm{ijk}$ & $57.4 \mathrm{abc}$ & 44.0efgh & Medium tolerant \\
\hline $\mathrm{CO} 448$ & $11.4 \mathrm{jk}$ & 52.3abce & 55.8abcdefg & Medium tolerant \\
\hline $\mathrm{CO} 437$ & $3.0 \mathrm{k}$ & 53.0abcd & $39.8 \mathrm{fgh}$ & Low tolerant \\
\hline $\mathrm{CO} 436$ & $1.2 \mathrm{k}$ & $2.9 \mathrm{~h}$ & $30.6 \mathrm{~h}$ & Low tolerant \\
\hline $\mathrm{CO} 440$ & $0.8 \mathrm{k}$ & $22.8 \mathrm{fgh}$ & $45.5 \mathrm{efgh}$ & Low tolerant \\
\hline
\end{tabular}

${ }^{\mathrm{z})}$ Means in the same column followed by different letters are significantly different according to Tukey's new multiple range test $(P<0.05)$.

Table 6. Correlation coefficients of under-studied traits at $5^{\circ} \mathrm{C}, 10^{\circ} \mathrm{C}, 23^{\circ} \mathrm{C}$ temperature condition.

\begin{tabular}{ccccc}
\hline \hline Temperature & Traits ${ }^{\mathrm{z})}$ & GR & GI & RL \\
\hline At $5^{\circ} \mathrm{C}$ & GR & & & \\
& GI & 0.90 & 0.64 & \\
& RL & 0.60 & 0.81 & 0.81 \\
At $10^{\circ} \mathrm{C}$ & SVI & 0.80 & & \\
& GR & & & \\
& GI & 0.98 & 0.30 & 0.83 \\
At $23^{\circ} \mathrm{C}$ & RV & 0.32 & 0.75 & \\
& GR & 0.75 & & 0.93 \\
\hline
\end{tabular}

${ }^{\text {z) }} P$-value $=0.05$.

observed in $\mathrm{CO} 440$ at $5^{\circ} \mathrm{C}$ treatment. At $10^{\circ} \mathrm{C}, \mathrm{CO} 444$ showed the strongest SVI, and CO436 showed the weakest SVI. At $23^{\circ} \mathrm{C}, \mathrm{CO} 447$ showed the highest value for SVI, and CO436 showed the weakest SVI. In our study, CO439,
CO438, CO450, CO435, and CO445 showed the best response in all given traits, while $\mathrm{CO} 437, \mathrm{CO} 436$, and CO440 showed the weakest response accordingly. 


\section{Seed germination pattems in different temperature conditions}

Supplementary Fig. 1 showed the distribution pattern for GR, GI, RL, and SVI investigated in 22 maize inbred lines under three temperature conditions $\left(5^{\circ} \mathrm{C}, 10^{\circ} \mathrm{C}\right.$, and $23^{\circ} \mathrm{C}$, respectively). A strong correlation was also found between GR and GI values under all temperature conditions $\left(5^{\circ} \mathrm{C}\right.$, $10^{\circ} \mathrm{C}$, and $23^{\circ} \mathrm{C}$ ); that is, with the increase in GR values there was a notable increase in GI as shown in Table 6. The analysis showed that least significant difference values among all studied traits were highly significant (that means all of the traits tested differed from all other traits).

The GR of maize lines CO439, CO438, CO450, and CO435 was found highly significant under all temperature conditions $(P<0.05)$. The $\mathrm{CO} 440$ showed the lowest $\mathrm{GR}$ at $5^{\circ} \mathrm{C}, \mathrm{CO} 436$ at $10^{\circ} \mathrm{C}$, and $\mathrm{CO} 443$ at $23^{\circ} \mathrm{C}$. Upon analyzing the GI of 22 maize inbred lines in different temperature conditions, the overall better performance was found in CO439, CO438, CO450, and CO435, while the lowest tolerant behavior was found to be the same for $\mathrm{CO} 437$, CO436, and CO440 inbred lines under all studied temperatures (Table 3).

When we look for the RL of 22 maize inbred lines in different temperature conditions, the performance was found significant for medium tolerant maize line (Table 4). The RL was found to be more significant with the GR under $5^{\circ} \mathrm{C}$ treatment. It was shown that under various temperature conditions, such as $5^{\circ} \mathrm{C}, 10^{\circ} \mathrm{C}$, and $23^{\circ} \mathrm{C}$, the different maize inbred lines $\mathrm{CO} 433, \mathrm{CO} 444$, and $\mathrm{CO} 449$ respectively showed significant mean values while no significant difference was found when analyzing the lowest tolerant lines as $\mathrm{CO} 436$ and $\mathrm{CO} 440$. For SVI, we found that it performed better under stress conditions $5^{\circ} \mathrm{C}(\mathrm{CO} 439)$, $10^{\circ} \mathrm{C}(\mathrm{CO} 444)$, and $23^{\circ} \mathrm{C}(\mathrm{CO} 445)$ (Table 5). The $\mathrm{CO} 436$ and $\mathrm{CO} 440$ showed the worst performance by giving the lowest significant mean values.

The overall performance of all the maize lines under different temperatures showed the significant behavior of better performing maize lines. SVI was also strongly correlated with GR under $5^{\circ} \mathrm{C}$ stress conditions. A similar finding was observed between GI and RL (strongly associated) under $5^{\circ} \mathrm{C}$ stress conditions (Table 6). The RL and SVI were strongly associated under all cold stress conditions (Table 6). Our correlation findings report that understudied lines performed better under $5^{\circ} \mathrm{C}$ cold stress as shown in Table 6, which clearly demonstrates that these lines have the potential to develop maize hybrids to increase grain yield under environmentally stressful conditions.

\section{DISCUSSION}

In many countries, maize crop has been adopted by sowing in early spring due to the low temperature of soil. Seeds can imbibe at chilling temperature, but often cannot germinate and may be attacked by fungi. In this study, we have observed the variance and significantly different values under three different cold temperature conditions. In results, some inbred lines were performed even at low compared with optimum temperature. It may be due to inbred lines at optimum temperature are more susceptible for disease. Table 2 demonstrates that the GR of seeds was mostly affected at all the studied temperature conditions. All lines gave higher rate because the mitochondria of plant cell are deeply affected due to low temperature during seedling growth. Furthermore, plants resist growing with an affected GR due to the seizing off of metabolic activities in plant cell (De Santis et al. 1999). Lovato et al. (2005) conducted a comparative experiment to check the relationship of seed emergence among germination tests. The standard cold temperature for maize is $10^{\circ} \mathrm{C}$ that may not be the favorable temperature in maize genotypes due to the hardness of cold temperature variance. Thus, they suggested that for the evaluation of cool tolerant genotypes, $5^{\circ} \mathrm{C}$ or $7.5^{\circ} \mathrm{C}$ is better to detect vigour difference among seed lots.

In a comparative result of GI, the values were the same as the GR from least significant different values in Table 3. This happened because the extremely low temperature reduced the germination and seedling growth as confirmed from the literature (Zheng et al. 2006). Wijewardana et al. (2015) also revealed similar results for cold tolerant corn hybrids. They reported that cold tolerant corn hybrids were useful to withstand harsh environmental conditions. 
Maximum yield can be approached in early season seeding and is more beneficial for breeders, as well as growers. An alternative cold test for maize germination was conducted by Matthews and Khajeh-Hosseini (2007). They suggested from their findings that quality of seed has great influence on seed vigour. Germination in older seeds can be delayed due to longer lag periods allow in seeds to repair metabolic and physiological activities (Matthews et al. 2011). Thus, the vigour of seed and seedling growth are also affected.

In our cold test result, RL values vary among lines (Table 4) not only affected by temperature stress, but also by seed quality and other metabolic activities during root growth. Plant roots are directly associated with suitable environment. At chilling temperature during water uptake, metabolic and biochemical activities are affected as the nutrient supply photosynthetic activities in crop plant and carbohydrate supply to shoot are directly related with root synthesis (Hund et al. 2008). Genotypes with high vigour must have the ability for root growth under freezing temperature. Higher root and shoot length are directly associated with seedling dry weight. Imran et al. (2013) also reported plant germination with increased ability to grow root under cold condition. Aroca et al. (2001) found in some maize root that hydraulic conductance activity seized at low temperature.

The embryo of seed is directly associated with vigourous seedling. In cereals, large grain promotes early vigour (Revilla et al. 1999). Higher vigour of crop plant is directly associated with higher yield. SVI values from our experiment were also affected at the lowest temperature value of $5^{\circ} \mathrm{C}$ (Table 5). The more precise assessment of physical and physiological seed lot performance can be done through vigour test. Kollipara et al. (2002) conducted an experiment to screen out recombinant inbred lines for cold germination and desiccation tolerance of phenotypes. From the results, it became easy to understand the gene involved in stress response during seed maturity and germination.

Rodríguez et al. (2010) conducted a research on maize in temperate areas at cold temperature. Among 95 screened members of the population, 11 exhibited best germination vigour under cold condition. At low temperature, a plant cell's metabolic, biochemical, and aerobic respiratory activities decrease. As a result, reactive oxidation species or plant growth retarding enzyme is produced, directly affecting plant germination and seedling vigour. However, for breeders and producers, the cultivar which shows the best performance against chilling temperature is highly recommended. To screen out stress tolerant cultivar is a challenging phenomenon in plant science. Thus, our findings will be helpful to carry out molecular design breeding in maize plant.

In conclusion, we can assume from the results of the cold germination test that at $5^{\circ} \mathrm{C}, 10^{\circ} \mathrm{C}$, and $23^{\circ} \mathrm{C}$, almost all 22 maize inbred lines show optimum germination and vigour. There is strong correlation between GR and index. In the overall comparison of studied traits, CO439, CO438, $\mathrm{CO} 450, \mathrm{CO} 435$, and $\mathrm{CO} 445$ show higher tolerance against all temperatures. Meanwhile, $\mathrm{CO} 437, \mathrm{CO} 436$, and CO440 lines perform weakly under the same circumstances. These selective high and low tolerant lines will be further investigated in molecular research and test crosses. This study also provides the basis for maize breeding research to assess best tolerant varieties under harsh conditions.

\section{ACKNOWLEDGEMENTS}

This study was supported by the Golden Seed Project (No. 213001-04-1-SBA10), the Ministry of Agriculture, Food, and Rural Affairs (MAFRA), the Ministry of Oceans and Fisheries (MOF), the Rural Development of Korea (RDA), and the Korea Forest Service (KFS).

\section{REFERENCES}

Abba EJ, Lovato A. 1999. Effect of seed storage temperature and relative humidity on maize (Zea mays L.) seed viability and vigour. Seed Sci. Tech. 27: 101-114.

Abdul-Baki AA, Anderson JD. 1973. Vigour determination in soybean seed by multiple criteria. J. Crop Sci. 13: 630-633.

Ali F, Ahsan M, Saeed NA, Ahmed M, Ali Q, Kanwal N, et al. 2014. Establishment and optimization of callus-to-plant regeneration system using mature and immature embryos 
of maize (Zea mays). Int. J. Agri. Biol. 16: 111-117.

Ali F, Kanwal N, Ahsan M, Ali Q, Bibi I, Niazi NK. 2015a. Multivariate analysis of grain yield and its attributing traits in different maize hybrids grown under heat and drought stress. Scientifica 2015: 1-6.

Ali F, Kanwal N, Ahsan M, Ali Q, Niazi NK. 2015b. Crop improvement through conventional and non-conventional breeding approaches for grain yield and quality traits in Zea mays. Life Sci. J. 12: 38-50.

Alvarez S, Roy Choudhury S, Pandey S. 2014. Comparative quantitative proteomics analysis of the ABA response of roots of drought-sensitive and drought-tolerant wheat varieties identifies proteomic signatures of drought adaptability. J. Proteome Res. 13: 1688-170.

Aroca R, Tognoni F, Irigoyen JJ, Díaz MS, Pardossi A. 2001. Different root low temperature response of two maize genotypes differing in chilling sensitivity. Plant Physiol. Biochem. 39: 1067-1073.

Bano S, Aslam M, Saleem M, Basra SMA, Aziz K. 2015. Evaluation of maize accessions under low temperature stress at early growth stages. J. Anim. Plant Sci. 25: 392-400.

DeVries M, Goggi AS, Moore KJ. 2007. Determining seed performance of frost damaged maize seed lots. Crop Sci. 47: 2089-2097.

De Santis A, Landi P, Genchi G. 1999. Changes of mitochondrial properties in maize seedlings associated with selection for germination at low temperature. Fatty acid composition, cytochrome $\mathrm{C}$ oxidase, and adenine nucleotide translocase activities. Plant Physiol. 119: 743-754.

Govender V, Aveling TAS, Kritzinger Q. 2008. The effect of traditional storage methods on germination and vigour of maize (Zea mays L.) from northern KwaZulu-Natal and southern Mozambique. S. Afr. J. Bot. 74: 190-196.

Guan YJ, Hu J, Wang XJ, Shao CX. 2009. Seed priming with chitosan improves maize germination and seedling growth in relation to physiological changes under low temperature stress. J. Zhejiang Uni. Sci. B. 10: 427-433.

Hoffman MA, Tranel DM, Hassen AT. 2015. Contribution of male inbreds to cold germination in maize hybrids. Seed Sci. Tech. 43: 197-207.

Hund A, Fracheboud Y, Soldati A, Stamp P. 2008. Cold tolerance of maize seedlings as determined by root morphology and photosynthetic traits. Eur. J. Agron. 28: 178-185.
Imran S, Afzal I, Basra SMA, Saqib M. 2013. Integrated seed priming with growth promoting substances enhances germination and seedling vigour of spring maize at low temperature. Int. J. Agric. Biol. 15: 1251-1257.

International Seed Testing Association (ISTA). 2002. International rules for seed testing. Seed Sci. Tech. 37: 54-59.

Kollipara KP, Saab IN, Wych RD, Lauer MJ, Singletary JW. 2002. Expression profiling of reciprocal maize hybrids divergent for cold germination and desiccation tolerance. Plant Physiol. 129: 974-992.

Lovato A, Noli E, Lovato AFS. 2005. The relationship between three cold test temperatures, accelerated ageing test and field emergence of maize seed. Seed Sci. Tech. 33: 249-253.

Matthews S, Beltrami E, El-Khadem R, Khajeh-Hosseini M, Nasehzadeh M, Urso G. 2011. Evidence that time for repair during early germination leads to vigour differences in maize. Seed Sci. Tech. 39: 501-509.

Matthews S, Khajeh-Hosseini M. 2007. Length of the lag period of germination and metabolic repair explain vigour differences in seed lots of maize (Zea mays). Seed Sci. Tech. 35: 200-212.

Mirosavljević M, Čanak P, Ćirić M, Nastasić A, Đukić D, Rajković M. 2013. Maize germination parameters and early seedlings growth under different levels of salt stress. Ratar. Povrt. 50: 49-53.

Revilla P, Butrón A, Malvar RA, Ordás RA. 1999. Relationship among kernel weight, early vigor, and growth in maize. Crop Sci. 39: 654-658.

Revilla P, Rodríguez VM, Ordás A, Rincent R, Charcosset A, Giauffret C, et al. 2014. Cold tolerance in two large maize inbred panels adapted to European climates. Crop Sci. 54: 1981-1991.

Riva-Roveda L, Périlleux C. 2015. Effects of cold temperatures on the early stages of maize (Zea mays L.). A review. Biotechnol. Agron. Soc. 19: 42-52.

Rodríguez VM, Romay MC, Ordás A, Revilla P. 2010. Evaluation of European maize (Zea mays L.) germplasm under cold conditions. Genet. Resour. Crop Evol. 57: 329-335.

Ruan S, Xue Q, Tylkowska K. 2002. The influence of priming on germination of rice (Oryza sativa L.) seeds and seedling emergence and performance in flooded soil. Seed Sci. Tech. 30: 61-67. 
Sanghera GS, Wani SH, Hussain W, Singh NB. 2011. Engineering cold stress tolerance in crop plants. Curr. Genomics 12: 30-43.

Tekrony DM. 2003. Precision is an essential component in seed vigour testing. Seed Sci. Tech. 31: 435-447.

TeKrony DM, Hunter JL. 1994. Effect of seed maturation and genotype on seed vigor in maize. Crop Sci. 35: 857-862.

Wijewardana C, Hock M, Henry B, Reddy KR. 2015.

Screening corn hybrids for cold tolerance using morphological traits for early-season seeding. Crop Sci. 55: 851-867.

Woltz J, TeKrony DM, Egli DB. 2006. Corn seed germination and vigor following freezing during seed development. Crop Sci. 46: 1526-1535.

Zheng Y, Hu J, Zhang S. 2006. Identification of chillingtolerance in maize inbred lines at germination and seedling growth stages. J. Zhejiang Univ. 32: 41-45. 